\title{
Temperature dependence of the frequency and noise of superconducting coplanar waveguide resonators
}

\author{
Shwetank Kumar, ${ }^{1}$ Jiansong Gao, ${ }^{1}$ Jonas Zmuidzinas, ${ }^{1, a)}$ Benjamin A. Mazin, ${ }^{1}$ \\ Henry G. LeDuc, ${ }^{2}$ and Peter K. Day ${ }^{2}$ \\ ${ }^{1}$ Division of Physics, Mathematics, and Astronomy California Institute of Technology, Pasadena, California \\ 91125, USA \\ ${ }^{2}$ Jet Propulsion Laboratory, California Institute of Technology, Pasadena, California 91109, USA
}

(Received 27 November 2007; accepted 19 February 2008; published online 25 March 2008)

\begin{abstract}
We present measurements of the temperature and power dependence of the resonance frequency and frequency noise of superconducting niobium thin-film coplanar waveguide resonators carried out at temperatures well below the superconducting transition $\left(T_{c}=9.2 \mathrm{~K}\right)$. The noise decreases by nearly two orders of magnitude as the temperature is increased from 120 to $1200 \mathrm{mK}$, while the variation of the resonance frequency with temperature over this range agrees well with the standard two-level system (TLS) model for amorphous dielectrics. These results support the hypothesis that TLSs are responsible for the noise in superconducting microresonators and have important implications for resonator applications such as qubits and photon detectors. (C) 2008 American Institute of Physics. [DOI: $10.1063 / 1.2894584]$
\end{abstract}

Superconducting microresonators are useful for photon detection, ${ }^{1-7}$ coupling to qubits, ${ }^{8-10}$ superconducting quantum interference device multiplexers, ${ }^{11,12}$ and studying basic physics. ${ }^{13-16}$ Such resonators have excess noise ${ }^{2,17}$ that is equivalent to a jitter of the resonance frequency, likely caused by two-level tunneling systems (TLSs) in amorphous dielectrics. ${ }^{18}$ Indeed, TLS models explain the unusual properties of amorphous materials at low temperatures, ${ }^{19-22}$ and recent qubit experiments ${ }^{23}$ have highlighted TLS effects in superconducting microcircuits. While the TLS energy splitting $\Delta E$ has a broad distribution, ${ }^{22}$ a resonator with frequency $f_{r}$ is most sensitive to TLS with $\Delta E \sim h f_{r}$. The level populations and relaxation rates of such TLS vary strongly at temperatures $T \sim h f_{r} / 2 k_{B}$, or around $100 \mathrm{mK}$ for the device studied here. Furthermore, such near-resonant TLS may saturate ${ }^{18}$ for strong resonator excitation power $P_{\mu w}$. Hence, measurements of the power and temperature variation of the resonator frequency and noise, as presented in this letter, provide a strong test of the TLS hypothesis.

We studied coplanar waveguide (CPW) quarterwavelength resonators ${ }^{2,18}$ fabricated on a high-resistivity $(\rho \geqslant 10 \mathrm{k} \Omega \mathrm{cm})$ crystalline silicon substrate by patterning a $200 \mathrm{~nm}$ thick niobium film using a photoresist mask and a $\mathrm{SF}_{6}$ inductively coupled plasma etch. In this device, TLS may be present in the native oxide surface layers on the metal film or substrate. ${ }^{18}$ The resonator is capacitively coupled to a CPW feedline (Fig. 1) that has a $10 \mu \mathrm{m}$ wide center strip and $6 \mu \mathrm{m}$ gaps between the center strip and the ground plane. For the resonator, these dimensions are 5 and $1 \mu \mathrm{m}$, respectively. The resonator length is $5.8 \mathrm{~mm}$, corresponding to $f_{r}=4.35 \mathrm{GHz}$. The coupling strength is set lithographically ${ }^{17,18}$ (see Fig. 1) and is characterized by the coupling-limited quality factor $Q_{c}=5 \times 10^{5}$.

The device was cooled using a dilution refrigerator, and its temperature was measured to $\pm 5 \mathrm{mK}$ accuracy using a calibrated $\mathrm{RuO}_{2}$ thermometer mounted on the copper sample enclosure. The microwave readout (Fig. 1) uses a standard

\footnotetext{
${ }^{a)}$ Electronic mail: jonas@caltech.edu.
}

$I Q$ homodyne mixing technique. ${ }^{2,17}$ The $I Q$ mixer's complex output voltage $\xi(f)=I(f)+j Q(f)$ follows a circular trajectory in the complex plane as the microwave excitation frequency $f$ is varied, ${ }^{18}$ and $f_{r}$ and $Q_{r}$ are determined by complex leastsquares fitting of this trajectory to a ten-parameter model:

$$
\begin{aligned}
\xi^{(\text {model })}(f)= & \left(B_{0}+B_{1} \delta x\right) \exp \left[i\left(\phi_{0}+\phi_{1} \delta x\right)\right] \\
& \times\left[\frac{S_{21}^{(r)}+2 j Q_{r} \delta x}{1+2 j Q_{r} \delta x}\right]+B_{2} \exp \left[i \phi_{2}\right] .
\end{aligned}
$$

Here $\delta x=\left(f-f_{r}\right) / f_{r}$ is the fractional frequency offset, $S_{21}^{(r)}$ is the complex forward transmission on resonance, $B_{0}+B_{1} \delta x$ allows for a linear gain variation, $\phi_{0}+\phi_{1} \delta x$ allows a similar linear phase variation, and $B_{2}$ and $\phi_{2}$ specify the output offset voltages of the $I Q$ mixer.

The combined noise of the resonator and readout electronics is measured by tuning the synthesizer to the reso-

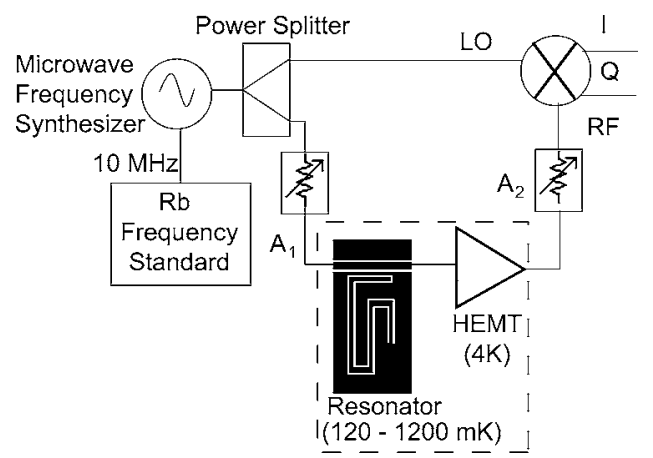

FIG. 1. The experimental setup is illustrated. The resonator (not to scale) is shown schematically; black represents the superconducting film; white represents bare substrate. The resonator is excited using a microwave synthesizer, and its output signal is sent to a cooled HEMT amplifier with $\sim 4 \mathrm{~K}$ noise temperature followed by a room-temperature amplifier. Amplitude and phase information are recovered simultaneously using an $I Q$ mixer. The attenuators $A_{1}$ and $A_{2}$ allow the incident microwave power $P_{\mu w}$ to be varied over a wide range while maintaining the optimal power level at the $I Q$ mixer's input by constraining the sum of the attenuations $A_{1}+A_{2}$ to be constant (in $\mathrm{dB}$ ). The $I Q$ output voltages are amplified, digitized, and recorded with 16 bit resolution at $250 \mathrm{ksample} / \mathrm{s}$. 


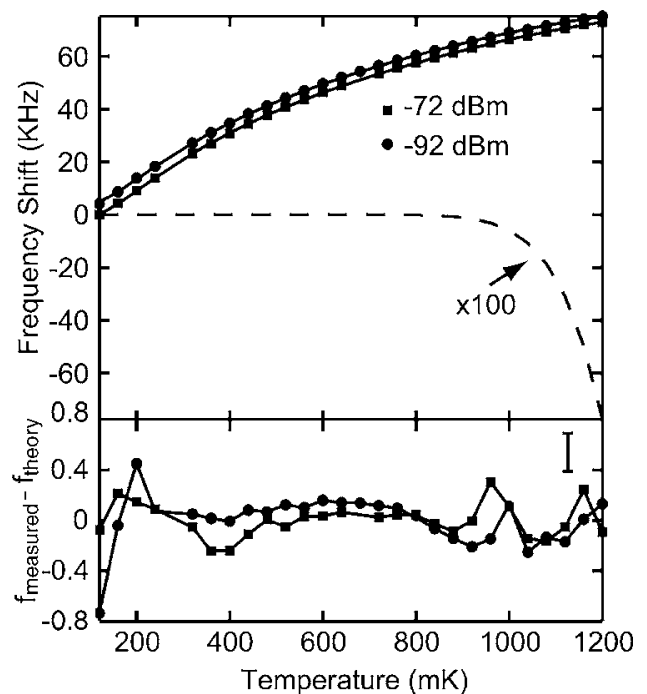

FIG. 2. (a) The resonance frequency shift, defined as $\delta f_{r}\left(T, P_{\mu w}\right)$ $=f_{r}\left(T, P_{\mu w}\right)-f_{r}(120 \mathrm{mK},-72 \mathrm{dBm})$, is plotted as a function of temperature for readout powers of $-72 \mathrm{dBm}$ (filled circle) and $-92 \mathrm{dBm}$ (filled square). The dashed line shows the frequency shift predicted by the Mattis-Bardeen theory but scaled up by a factor of 100 . The solid lines represent fits to the data using Eq. (2). (b) A plot of the residuals after subtracting the fit from the data; the representative error bar indicates that the fit matches the data to within the $\pm 5 \mathrm{mK}$ accuracy of the thermometry.

nance $\left(f=f_{r}\right)$ and digitizing the fluctuations $\delta \xi(t) .^{2,17,18}$ The noise analysis follows our previous work. ${ }^{18}$ In brief, the noise covariance matrix is calculated and diagonalized at each noise frequency, yielding power spectra for amplitude (dissipation) and phase (frequency) fluctuations. The amplitude noise is consistent with the electronics noise floor measured off resonance, so we estimate the resonator's frequency noise by subtracting the amplitude noise spectrum from the phase noise spectrum.

The quality factor $Q_{r}$ lies in the range of (1.5-4.5) $\times 10^{5}$ and is both temperature and power dependent; however, the interpretation is not straightforward due to TLS saturation effects. ${ }^{18,23,24}$ In contrast, $f_{r}$ is much less affected by TLS saturation. ${ }^{24}$ Figure 2 (a) shows the measured temperature dependence of the frequency shift $\delta f_{r}\left(T, P_{\mu w}\right)$. Note that $\delta f_{r}$ increases with temperature, whereas the MattisBardeen theory ${ }^{25}$ predicts a superconductivity-related frequency shift that is much smaller and opposite in sign. Instead, the data fit quite well to the functional form predicted TLS theory. ${ }^{22}$ Above $900 \mathrm{mK}$, this fit can be further improved by including the Mattis-Bardeen contribution according to the following model:

$$
\begin{aligned}
\frac{\delta f_{r}^{(\text {model })}\left(T, P_{\mu w}\right)}{f_{r}}= & C_{1}\left(P_{\mu w}\right)+C_{2}\left(P_{\mu w}\right) \\
& \times\left[\operatorname{Re} \psi\left(\frac{1}{2}+\frac{h f_{r}}{2 \pi i k_{b} T}\right)-\log \left(\frac{h f_{r}}{k_{b} T}\right)\right] \\
& +\frac{C_{3}}{4}\left[\frac{\sigma_{2}(T)-\sigma_{2}(0)}{\sigma_{2}(0)}\right]
\end{aligned}
$$

There are three free parameters for each power level: $C_{1}\left(P_{\mu w}\right)$ allows a small power-dependent frequency shift relative to $f_{r}(120 \mathrm{mK},-72 \mathrm{dBm}) ; C_{2}\left(P_{\mu w}\right)$ is the coefficient of the TLS linear response term ${ }^{26}$ and is allowed to vary with power to account for possible TLS saturation; $C_{3}$ is the kinetic inductance fraction of the CPW line ${ }^{27}$ and should be

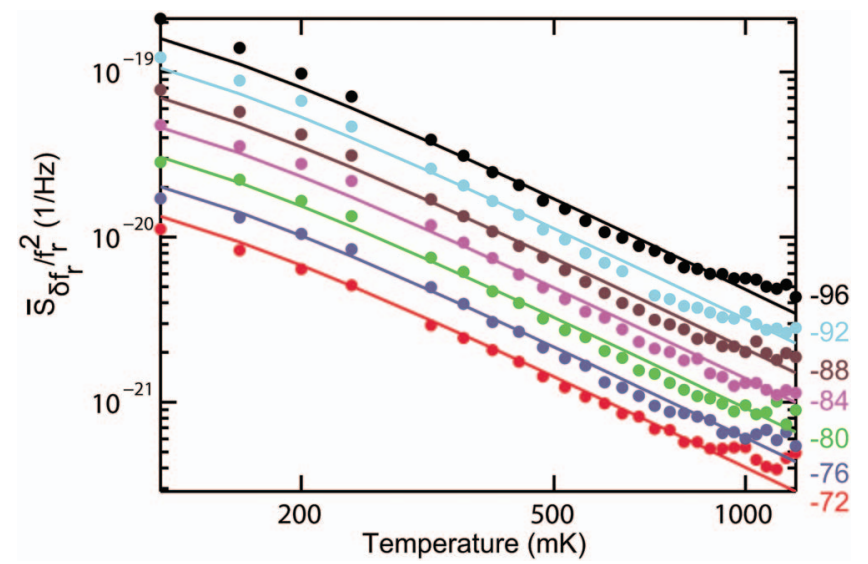

FIG. 3. (Color) The average value of the fractional frequency noise power spectrum in the $200-300 \mathrm{~Hz}$ range $\left[\bar{S}_{\delta f_{r}}(\nu) / f_{r}^{2}\right]$ is plotted as a function of temperature $(T)$ for several values of the microwave readout power $\left(P_{\mu w}\right)$. The power levels $P_{\mu w}$ range from -96 to $-72 \mathrm{dBm}$, as indicated by the labels on the right. The colored lines show the fit to Eq. (3).

constant over the range of readout power used. The data were fitted for readout power values from -72 to $-92 \mathrm{dBm}$ in steps of $4 \mathrm{dBm}$. The value of $C_{3}$ was indeed found to be constant $\left(C_{3}=0.104 \pm 0.021\right)$ and in close agreement with the expected value, ${ }^{27} C_{3}=0.125$. Meanwhile, $C_{1}=(1.948 \pm 0.002) \times 10^{-5}$ at $-72 \mathrm{dBm}$ and $(1.902 \pm 0.002)$ $\times 10^{-5}$ at $-92 \mathrm{dBm}$, while $C_{2}=(9.09 \pm 0.02) \times 10^{-6}$ and $(9.39 \pm 0.02) \times 10^{-6}$ for -72 and $-92 \mathrm{dBm}$, respectively. For a fixed temperature $T$, we find that the frequency shift $\delta f_{r}$ scales with power approximately as $P_{\text {int }}^{0.3}$, where $P_{\text {int }}$ $=2 Q_{r}^{2} P_{\mu w} /\left(\pi Q_{c}\right)$ is the resonator's internal microwave power.

The coefficient $C_{2}$ is a measure of the number of TLSs that are coupled to the resonator's electric field $\vec{E}(\vec{r})$. This relationship may be quantified in terms of the microwave loss tangent $\delta$ of the amorphous TLS material and the $|\vec{E}|^{2}$-weighted volume filling fraction $F$ of that material according to $C_{2}=F \delta / \pi$. Typical amorphous materials have loss tangents of order $\delta \sim 10^{-2}-10^{-3}$. Assuming a uniform distribution of TLS on the surface of the resonator, perhaps due to surface oxides, a reasonable oxide thickness of order of $10 \mathrm{~nm}$ would be consistent with the observed filling factor $F \sim 10^{-2}$.

The temperature and power dependence of the resonator noise was quantified by first calculating the fractional frequency noise spectrum, ${ }^{18} S_{\delta f_{r}}(\nu) / f_{r}^{2}$, which was then averaged over the range of $200-300 \mathrm{~Hz}$, a clean portion of the spectrum well above the high electron mobility transistor (HEMT) noise floor at low temperatures. The resulting values are plotted in Fig. 3, demonstrating the very strong temperature dependence of noise. These data may be described reasonably well by the fitting function

$$
\frac{\bar{S}_{\delta f_{r}}(\nu)}{f_{r}^{2}}=A P_{\mu w}^{\alpha} T^{\beta} \tanh ^{2}\left(\frac{h f_{r}}{2 k_{B} T}\right),
$$

with indices $\alpha=-0.46 \pm 0.005$ and $\beta=-0.14 \pm 0.02$. The value of $\alpha$ is consistent with previous work. ${ }^{18}$ While the inclusion of the hyperbolic tangent factor is motivated by a particular model for the TLS noise ${ }^{24}$ and the low value of $\beta$ is suggestive, at present, the data cannot distinguish between this model and a simple power law temperature dependence AIP license or copyright; see http://apl.aip.org/apl/copyright.jsp 
of the form $T^{-1.73}$. Additional measurements, especially at lower temperatures, will be needed to further elucidate the physical mechanism of the TLS noise.

In conclusion, both the frequency and noise of our superconducting CPW resonators show substantial variation at temperatures far below the superconducting critical temperature. The variation of the resonance frequency is well described by TLS theory with plausible values for the loss tangent and filling factor. Combined, these results strongly suggest that the resonator noise is also due to TLS and is not related to the superconductor. The temperature dependence of the noise also has important practical implications. For instance, if the TLS origin of the noise is correct, designing resonators to operate in the regime $f_{r} \ll 2 k T / h$ could result in lower noise and improved performance.

We thank Sunil Golwala, Kent Irwin, Andrew Lange, Konrad Lehnert, Harvey Moseley, and especially John Martinis for useful discussions. This work was supported in part by the NASA Science Mission Directorate, JPL, and the Gordon and Betty Moore Foundation.

${ }^{1}$ B. A. Mazin, P. K. Day, J. Zmuidzinas, and H. G. Leduc, AIP Conf. Proc. 605, 309 (2002)

${ }^{2}$ P. K. Day, H. G. LeDuc, B. A. Mazin, A. Vayonakis, and J. Zmuidzinas, Nature (London) 425, 817 (2003).

${ }^{3}$ J. Baselmans, S. J. C. Yates, R. Barends, Y. J. Y. Lankwarden, J. R. Gao, H. Hoevers, and T. M. Klapwijk, J. Low Temp. Phys. 151, 524 (2008).

${ }^{4}$ G. Vardulakis, S. Withington, D. J. Goldie, and D. M. Glowacka, Meas. Sci. Technol. 19, 015509 (2008).

${ }^{5}$ D. R. Schmidt, K. W. Lehnert, A. M. Clark, W. D. Duncan, K. D. Irwin, N. Miller, and J. N. Ullom, Appl. Phys. Lett. 86, 053505 (2005).

${ }^{6}$ B. A. Mazin, M. E. Eckart, B. Bumble, S. Golwala, P. K. Day, J. Zmuidzinas, and F. A. Harrison, Appl. Phys. Lett. 89, 222507 (2006).
${ }^{7}$ R. Barends, J. Baselmans, J. Hovenier, J. Gao, S. Yates, T. Klapwijk, and H. Hoevers, IEEE Trans. Appl. Supercond. 17, 263 (2007).

${ }^{8}$ A. Wallraff, D. I. Schuster, A. Blais, L. Frunzio, R.-S. Huang, J. Majer, S. Kumar, S. M. Girvin, and R. J. Schoelkopf, Nature (London) 431, 162 (2004).

${ }^{9}$ J. Lee, W. Oliver, T. Orlando, and K. Berggren, IEEE Trans. Appl. Supercond. 15, 841 (2005).

${ }^{10}$ M. Sillanpaa, J. Park, and R. Simmonds, Nature (London) 449, 438 (2007).

${ }^{11}$ K. D. Irwin and K. W. Lehnert, Appl. Phys. Lett. 85, 2107 (2004).

${ }^{12}$ K. Lehnert, K. Irwin, M. Castellanos-Beltran, J. Mates, and L. Vale, IEEE Trans. Appl. Supercond. 17, 705 (2007).

${ }^{13}$ B. Abdo, E. Arbel-Segev, O. Shtempluck, and E. Buks, J. Appl. Phys. 101, 083909 (2007).

${ }^{14}$ B. Yurke and E. Buks, J. Lightwave Technol. 24, 5054 (2006).

${ }^{15}$ E. Abrel-Segev, B. Abdo, O. Shtempluck, E. Buks, and B. Yurke, Phys. Lett. A 370, 202 (2007).

${ }^{16}$ E. A. Tholn, A. Ergl, E. M. Doherty, F. M. Weber, F. Grgis, and D. B. Haviland, Appl. Phys. Lett. 90, 253509 (2007).

${ }^{17}$ B. A. Mazin, Ph. D. thesis, Caltech, 2004.

${ }^{18}$ J. Gao, J. Zmuidzinas, B. A. Mazin, H. G. LeDuc, and P. K. Day, Appl. Phys. Lett. 90, 102507 (2007).

${ }^{19}$ S. Hunklinger, W. Arnold, S. Stein, R. Nava, and K. Dransfeld, Phys. Lett. 42, 253 (1972).

${ }^{20}$ M. von Schickfus, S. Hunklinger, and L. Pich, Phys. Rev. Lett. 35, 876 (1975).

${ }^{21}$ B. D. Laikhtman, Phys. Rev. B 31, 490 (1985).

${ }^{22}$ W. A. Phillips, Rep. Prog. Phys. 50, 1657 (1987).

${ }^{23}$ J. M. Martinis, K. B. Cooper, R. McDermott, M. Steffen, M. Ansmann, K. D. Osborn, K. Cicak, S. Oh, D. P. Pappas, R. W. Simmonds, and C. C. Yu, Phys. Rev. Lett. 95, 210503 (2005).

${ }^{24}$ J. Martinis, personal communication (April 14, 2007 and September 20, 2007).

${ }^{25}$ D. C. Mattis and J. Bardeen, Phys. Rev. 111, 412 (1958).

${ }^{26} \mathrm{~W}$. Mason and R. Thurston, Physical Acoustics: Principles and Methods (Academic, New York, 1976), Vol. 12.

${ }^{27}$ J. Gao, J. Zmuidzinas, B. A. Mazin, P. K. Day, and H. G. Leduc, Nucl. Instrum. Methods Phys. Res. A 559, 585 (2006). 